Pacific

Journal of

Mathematics

GRAPHS, DETERMINANTS OF KNOTS AND HYPERBOLIC VOLUME

AleXANDER StOIMENOW

Volume 232

No. 2

October 2007 


\title{
GRAPHS, DETERMINANTS OF KNOTS AND HYPERBOLIC VOLUME
}

\author{
ALEXANDER STOIMENOW
}

\begin{abstract}
Using estimates for the number of spanning trees of planar graphs, we prove inequalities for the determinant of alternating links in terms of their hyperbolic volume.
\end{abstract}

\section{Introduction}

If $\Delta_{L}$ denotes the (1-variable) Alexander polynomial [1928] of a link $L$ in $S^{3}$, then $\operatorname{det} L=\left|\Delta_{L}(-1)\right|$ is the order of the homology group $H_{1}\left(D_{L}\right)$ (over $\mathbb{Z}$ ) of the double branched cover $D_{L}$ of $S^{3}$ over $L$ (or 0 if this group is infinite). This number is often called the determinant of $L$ because of its expression (up to sign) as the determinant of a Seifert matrix [Rolfsen 1976, page 213] or a Goeritz matrix [Gordon and Litherland 1978]. The group $H_{1}\left(D_{L}\right)$ carries much interesting information on the link (in particular conditions for sliceness [Rolfsen 1976] and achirality [Hartley and Kawauchi 1979; Stoimenow 2005], and unknotting number estimates [Wendt 1937]). The determinant can be alternatively evaluated from the Jones polynomial $V[1985]$ as $|V(-1)|$, and for alternating diagrams it has a nice combinatorial interpretation (see [Krebes 1999]) in terms of the Kauffman state model [1987].

Based on experimental evidence, Dunfield [2000] proposed interesting relations (though he did not state them very precisely) between the determinant and the hyperbolic volume of (complements in $S^{3}$ of) alternating knots and links. Roughly speaking, he found that the determinant should have upper and lower bounds which are exponential in terms of the volume. The main aim of this paper is to prove rigorous versions of Dunfield's conjectured inequalities. To formulate them, we will denote by $c(L)$ the crossing number of a link $L$, and by vol $L$ the hyperbolic volume of (the complement in $S^{3}$ of) $L$, or 0 if $L$ is not hyperbolic.

Theorem 1.1. If $L$ is a nontrivial nonsplit alternating link, then

$$
\operatorname{det} L \geq 2 \cdot 1.0355^{\mathrm{vol} L} \text {. }
$$

MSC2000: primary 57M25; secondary 05C05, 05C62, 57M12, 57M50.

Keywords: alternating knot, determinant, spanning tree, planar graph, hyperbolic volume.

Supported by the 21 st Century COE Program. 
Theorem 1.2. There are constants $C_{1}, C_{2}>0$ such that, for any hyperbolic alternating link $L$,

$$
\operatorname{det} L \leq\left(\frac{C_{1} \cdot c(L)}{\operatorname{vol} L}\right)^{C_{2} \operatorname{vol} L}
$$

It is natural (as in [Dunfield 2000]) to regard such statements also as a relation between the Jones polynomial and hyperbolic volume. The motivation for such a viewpoint lies in the variety of connections that has recently become apparent between the volume and Jones-type invariants.

One such connection worth remarking on is the Volume conjecture [Murakami and Murakami 2001], which asserts that one can exactly determine the volume of a link from the Jones polynomial and all its cables. (The conjecture is of theoretical rather than practical importance, since the volume evaluation it proposes is far too expensive.) In another venue, Khovanov suggested an extension of Dunfield's correspondence to nonalternating links, replacing the determinant by the total dimension of his homology [Khovanov 2000] generalizing the Jones polynomial. (This is more closely related to our result, but still outside our scope.)

There is also accumulating evidence that the (ordinary) Jones polynomial might be able to provide way practical bounds on the volume in a different way. Such bounds, which involve degrees or coefficients of the polynomial, have been obtained for alternating links by Dasbach and Lin [2007] and for Montesinos and 3-braid links by myself [Stoimenow 2006]. Contrarily, the question whether one can increase the hyperbolic volume without bound while preserving the Jones polynomial (or at least its Mahler measure) remains open.

Theorems 1.1 and 1.2 improve on closely related, but unpublished, work of Chris Leininger and Ilya Kofman. They seem qualitatively close to the optimum, apart from the determination and improvement of constants. Both theorems apply the twist number of link diagrams. Theorem 1.2 is not hard to deduce (with constants $C_{1}, C_{2}$ which are easy to make explicit; see its proof in Section 4). It requires a recent lower bound for the volume of alternating links, first obtained by Lackenby [2004] and later, with an improved constant, by Agol, Storm and W. Thurston [Agol et al. 2007].

Theorem 1.1, although similar in nature, requires considerably more effort, and it is the main content of this paper. Our proof of it builds most decisively on the useful relationship between knot and graph theory, stating that the determinant of an alternating diagram is the number of spanning trees of a certain planar (checkerboard) graph associated to this diagram. This relationship is well known and was used already decades ago by Crowell [1959]. However, the only general lower estimates on the determinant derived from it so far have been linear in the crossing number. We need to show that we can make the linear bound exponential, if we 
replace the crossing number by the twist number (Theorem 4.3). This occupies a large part of the paper.

Our work can be considered mostly independent from [Lackenby 2004], because the upper volume bounds follow from previously known classical facts about hyperbolic volume (see for example [Brittenham 1998]). It is only the optimal choice of constants, due to I. Agol and D. Thurston [2004], that goes into Theorem 1.1 for a better estimate. The same is true of Proposition 6.3, which uses the proof of Theorem 1.1 to improve the inequality in the special case of arborescent (Conwayalgebraic) links.

Other work relating the volume to the Alexander polynomial has been done by Kalfagianni [2004] and Silver and Whitten [2005], and to the Jones polynomial by Silver, Williams and myself [Silver et al. 2006]. The growth of the order of the homology groups of the higher order cyclic branched covers of $S^{3}$ over a fixed link $L$ has been studied by Gordon [1972], and later by González-Acuña and Short [1991] and Riley [1990]. These numbers can still be determined from the Alexander polynomial, but lack a nice combinatorial description, and are rather of number-theoretical interest. (Riley's growth estimates in fact use some deep tools from number theory.) Their growth rate is related to questions on entropy and Mahler measure.

\section{The determinant and twist number of alternating diagrams}

Before we come to the proof of our results, we will briefly review the necessary, and previously initiated, framework to incorporate them into.

By means of the relation $\Delta(-1)=V(-1)$ to the Jones polynomial (see [Jones 1987, §12]) the determinant provides a bridge between the classical Alexander polynomial and its modern successors [Brandt et al. 1986; Freyd et al. 1985; Kauffman 1990; Jones 1985], whose nature is largely combinatorial. It is part of the small amount of topologically understandable information encoded in these more recent invariants.

At the same time, this relation opens combinatorial approaches for calculating the determinant. One such approach, which is particularly nice for alternating diagrams, was given in [Krebes 1999] using the Kauffman bracket/state model for the Jones polynomial.

If $D$ is an alternating link diagram, consider $\hat{D} \subset \mathbb{R}^{2}$, the (image of) the associated immersed plane curve(s). Then $\operatorname{det} D$ is the number of ways to splice the crossings (self-intersections) of $\hat{D}$, as in (3), so that the resulting collection of

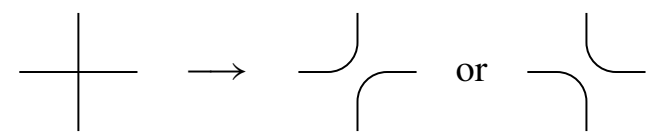




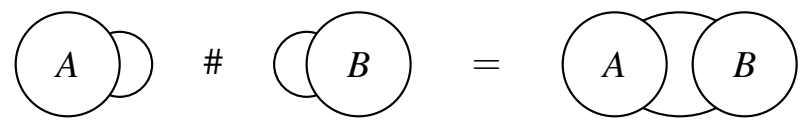

Figure 1. The connected sum.

disjoint circles has only one component (a single circle); such choices of splicings are called monocyclic states in [Krebes 1999].

Definition 2.1. The diagram on the right in Figure 1 is called the connected sum $A \# B$ of the diagrams $A$ and $B$. If a diagram $D$ can be represented as the connected sum of diagrams $A$ and $B$ having each at least one crossing, $D$ is called composite; otherwise it is called prime. Equivalently, a diagram is prime if any closed curve intersecting it in exactly two points does not contain a crossing in one of its interior or exterior.

Any diagram $D$ can be written as $D_{1} \# D_{2} \# \cdots \# D_{n}$ in such a way that all the $D_{i}$ are prime and have at least one crossing. The $D_{i}$ are called the prime factors of $D$. A link $L$ is prime if, for any composite diagram $D_{1} \# D_{2}$ of $L$, exactly one of $D_{1}, D_{2}$ is an unknot diagram. (Thus the unknot is not prime per convention, though its 0 -crossing diagram is prime.)

Definition 2.2. A diagram $D$ is split, or disconnected, if there is a closed curve which does not intersect $D$, but which contains parts of $D$ in both its interior and exterior. Otherwise $D$ is called connected. A link is split if it has a split diagram.

Thus a diagram $D$ is connected if and only if its plane curve $\hat{D}$ is a connected set. From the explanation above, then, we see that an alternating diagram has nonzero determinant if and only if it is connected.

Definition 2.3. A crossing $q$ in a link diagram $D$ is called nugatory if there is a closed (smooth) plane curve intersecting $D$ transversely in $q$ and nowhere else. A diagram is called reduced if it has no nugatory crossings.

Theorem 2.4 [Menasco 1984]. An alternating reduced diagram of a link L is prime if and only if $L$ is prime.

Definition 2.5. Let $c(D)$ be the crossing number of a link diagram $D$. Let $c(L)$ be the crossing number of a link $L$, that is, the minimal crossing number of all diagrams $D$ of $L$. A link $L$ is trivial if $c(L)=0$.

Theorem 2.6 [Kauffman 1987; Murasugi 1987; Thistlethwaite 1987]. Every alternating reduced diagram is of minimal crossing number for the link it represents.

To explain the Lackenby-Agol-Thurston inequalities, we introduce the notion of twist equivalence of crossings. The version of this relation we present here is slightly different from that of [Lackenby 2004]. First we introduce a diagram move called the flype, shown in (4): 

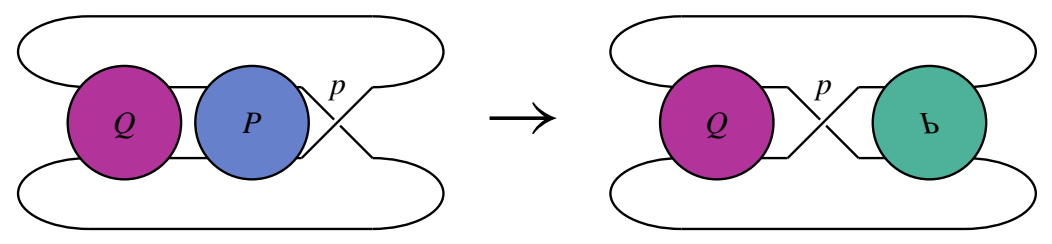

A clasp $\bigcirc$ is a tangle consisting of two crossings, that is, a pair of crossings that bound a bigonal (2-corner) region in the plane complement of a diagram.

Definition 2.7. Let $D$ be a reduced link diagram. We say that two crossings $p$ and $q$ of $D$ are twist equivalent if there exists a flype making $p$ and $q$ into a clasp.

(5)

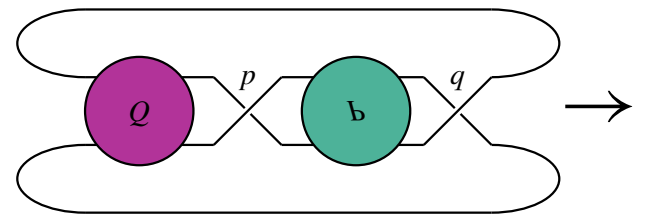

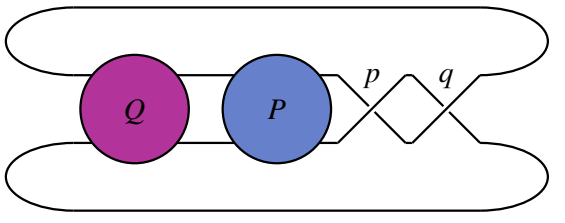

(This formulation assumes the obvious correspondence between the crossings in the diagrams on both sides of (4).) We call this relation a twist equivalence. Let $t(D)$ be the twist number of a diagram $D$, that is, the number of its twist equivalence classes. The twist number $t_{\min }(K)$ of a knot or link $K$ is the minimal twist number of any diagram $D$ of $K$.

For knot diagrams an alternative definition of twist equivalence, which follows its independently studied variants in [Stoimenow 2001; 2003], goes thus: Call two crossings $p, q$ in a knot diagram linked if their crossing points are passed in cyclic order $p q p q$ (rather than $p p q q$ ) along the diagram plane curve. Then $p$ and $q$ are twist equivalent if and only if each crossing $r \neq p, q$ is linked with $p$ exactly if it is linked with $q$.

Menasco and Thistlethwaite [1991; 1993] proved that alternating diagrams of the same link are related by flypes. Since our definition of twist equivalence is invariant under flypes, we see that the twist number is independent of the choice of alternating diagram. Hence we can say:

Definition 2.8. For an alternating link $L$, define the twist number of $L$ as $t(L)=$ $t(D)$, where $D$ is some alternating diagram of $L$.

For an alternating knot the obvious inequality $t_{\min }(K) \leq t(K)$ may be strict; an easy example is the knot $7_{4}$. Thus there are alternating knots with nonalternating diagrams of smaller twist number than the twist number of their alternating diagram(s). In particular, the minimal twist number and the minimal crossing number (see Theorem 2.6) may not be simultaneously attainable by a diagram.

Our notion of twist equivalence is slightly more relaxed than Lackenby's [2004], the difference being that he did not allow flypes. We call Lackenby's equivalence 
strong twist equivalence. It has been repeatedly observed that by means flypes all twist equivalent crossings can be made strongly twist equivalent (and (5) indicates how); Lackenby formulated this fact as the existence of twist reduced diagrams. Thus we can work with twist equivalence in our sense as with twist equivalence in Lackenby's sense (or strong twist equivalence in our sense), provided we can assume that the alternating diagram is twist reduced. With this remark, we can state the inequalities (into which work of Agol, Lackenby, Storm, D. Thurston and W. Thurston goes in) as follows:

Theorem 2.9 [Lackenby 2004; Agol et al. 2007]. For a nontrivial prime alternating link $L$, we have

$$
10 V_{0}(t(L)-1) \geq \operatorname{vol} L \geq V_{8}(t(L)-2),
$$

where $V_{0}=\operatorname{vol}\left(4_{1}\right) / 2 \approx 1.01494$ is the volume of the ideal tetrahedron, and $V_{8} \approx$ 1.83193 .

Both constants are optimal; the 10 on the left is approximated asymptotically by links in a certain iterative pattern, described in [Agol and Thurston 2004]. The constant $V_{8}$ on the right makes the inequality sharp for the Borromean rings.

\section{Graphs}

General preliminaries. Later on it will be helpful for us to translate the statements we would like to prove about link diagrams into statements about planar graphs. As graphs occur throughout the paper, we set up some related terminology, most of which (like dual graph, deleting or removing edges, etc.) is standard, and also explained in [Murasugi and Stoimenow 2003]. For clarification, we repeat some of these definitions, and collect several simple facts that will be used later. Some new notions seem also necessary to introduce.

Definition 3.1. A loop edge is an edge connecting a vertex with itself. A multiple $e d g e e$ is the set of all $n$ edges connecting the same pair of (distinct) vertices; we assume $n \geq 2$. The number $n$ is the multiplicity of $e$. Edges that belong to the same multiple edge (that is, connect the same 2 vertices) are called parallel. A graph is called simple if it has no multiple edges, and loop-free if it has no loop edges.

Definition 3.2. For a graph $G$, let $E(G)$ the set of edges and $e(G)$ the number of edges. An edge of multiplicity $n$ is understood to contribute $n$ to $e(G)$. We call $G$ trivial if $e(G)=0$. An edge between vertices $v$ and $w$, which is not given a separate name, will be written as $v w$.

We will assume that graphs are connected and have no loop edges and vertices of valence 1 , unless the opposite is clear. 
Definition 3.3. A cycle-free graph is a forest. A connected forest is a tree. If $G$ is a connected graph of $n$ vertices, a spanning tree of $G$ is a tree contained in the edges of $G$, which is maximal, that is, has $n-1$ edges. For a graph $G$, we denote by $s(G)$ the number of spanning trees $G$ (or 0 if $G$ is not connected).

Definition 3.4. If $G$ is a graph, a set of edges $\left\{e_{1}, \ldots, e_{n}\right\}$ of $G$ is an $n$-cut (or simply a cut) if the subgraph obtained by removing $e_{1}, \ldots, e_{n}$ is disconnected. A 1 -cut is an isthmus. We say that $G$ is $n$-connected if it has no $k$-cut for $k<n$. If $G$ has more than one vertex, the set of all edges incident to a vertex forms a cut. A cut vertex is a vertex for which the subgraph obtained by removing it (and all incident edges) is disconnected.

Definition 3.5. The join $G=G_{1} * G_{2}$ of two graphs $G_{1}$ and $G_{2}$ is a graph $G$ obtained by identifying a vertex in $G_{1}$ with a vertex in $G_{2}$, called the join vertices. The new vertex is a cut vertex of $G$. (The choice of join vertices has an effect on $G$, but this choice is irrelevant for our purposes.) Every nontrivial graph $G$ can be written as a join $G_{1} * \cdots * G_{n}$ for some nontrivial graphs $G_{i}$ such that no $G_{i}$ has a cut vertex. We call the $G_{i}$ the join factors of the graph $G$. If the decomposition is nontrivial (that is, if $n>1$ ), we call $G$ decomposable.

By definition, a graph is 2-connected if and only if it has no isthmus. The following is an easy observation:

Lemma 3.6. $G_{1} * G_{2}$ is 2-connected if and only if $G_{1}$ and $G_{2}$ are so. $G_{1} * G_{2}$ has an isthmus or loop edge if and only if one of $G_{1}$ or $G_{2}$ has one.

Definition 3.7. A graph $G$ is planar if it is embedded in the plane. For each $n$ cut $\left\{e_{1}, \ldots, e_{n}\right\}$ of a planar graph $G$ one can draw a closed curve in the plane, intersecting $G$ transversely only in (single interior points of) the edges $e_{1}, \ldots, e_{n}$. We call such curve a cut curve or circuit in $G$. Clearly the curve of a cut determines the cut uniquely.

Definition 3.8. Assume $G$ is a planar graph. A region $E$ of $G$ is any connected component of the complement of $G$. Its boundary is denoted $\partial E$ and understood as a set of edges of $G$. If an edge $e$ of $G$ lies in the boundary of regions $A$ and $B$, we say that $A$ is opposite to $B$ at $e$, or that $A$ and $B$ are opposite.

Thus $G$ is 2-connected if it is connected and no region is opposite to itself at some edge. Also, $G$ is 3-connected if it is 2 -connected and for any two regions there is at most one edge at which they are opposite.

The join, defined so far for abstract graphs, can naturally be extended to planar graphs. (The ambiguity in the choice of vertices carries over, and a new ambiguity arises from the planar embedding; but again this will not be a problem here.)

Definition 3.9. The dual graph of a planar graph $G$ is denoted by $G^{*}$. There is a natural bijection of edges between $G$ and $G^{*}$; in that sense we can talk of the dual 
$e^{*} \in E\left(G^{*}\right)$ of an edge $e \in E(G)$. Similarly the dual $A^{*}$ of a region $A$ of $G$ is a vertex of $G^{*}$. We consider $G^{*}$ embedded in the plane in the obvious way: an edge $e \in E(G)$ and its dual $e^{*}$ meet exactly once (and transversely), and other edges do not meet at all.

We have the following simple properties of the dual:

Lemma 3.10. For planar graphs $G_{1}$ and $G_{2}$, and a proper choice of join vertices, $\left(G_{1} * G_{2}\right)^{*}=G_{1}^{*} * G_{2}^{*}$.

Lemma 3.11. Let $G$ be a planar graph.

(i) An edge e of $G$ is an isthmus if and only if the edge $e^{*}$ in $G^{*}$ is a loop edge.

(ii) $G$ has a cut vertex if and only if $G^{*}$ has one.

(iii) If $G$ has no isthmus or loop edge, then $G$ is 3-connected if and only if $G^{*}$ is simple.

Checkerboard graphs. To any alternating link diagram $D$ one can associate its checkerboard graph $G=\Gamma(D)$ in the following manner (see Figure 2, or also [Kauffman 1990; Dasbach and Hougardy 1996; Krebes 1999; Stoimenow 2005; Thistlethwaite 1988]). Consider the plane curve $\hat{D}$ of $D$ as a 4-valent planar graph. There is a black-white coloring of the regions of $\hat{D}$, the checkerboard coloring, which assigns different colors to each pair of regions opposite at some edge of $\hat{D}$. Then $\Gamma(D)$ is defined to have a vertex for each black region of $\hat{D}$ and an edge for each crossing of $D$ as follows:

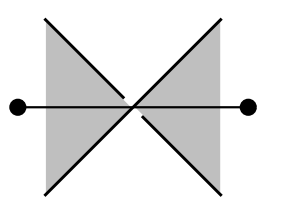

or

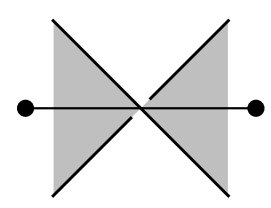

Assuming $D$ is connected, there are only two choices of checkerboard colorings (equivalent up to the interchange of colors) and so $\Gamma(D)$ is uniquely defined up to duality, and duality corresponds to interchanging the colors.

Thus the checkerboard graph is a planar graph, possibly with multiple edges. Conversely, any such graph is the checkerboard graph of some link diagram. A diagram $D$ is alternating if for all crossings the same one of the two local pictures in (6) occurs. Thus the checkerboard graph determines an alternating diagram up to mirroring. The following is easy to see:

Lemma 3.12. The checkerboard graph $G=\Gamma(D)$ of a diagram $D$ has no cut vertex if and only if $D$ is prime. More precisely, the connected sum of diagrams corresponds to the join of checkerboard graphs:

$$
\Gamma\left(D_{1} \# D_{2}\right)=\Gamma\left(D_{1}\right) * \Gamma\left(D_{2}\right) .
$$



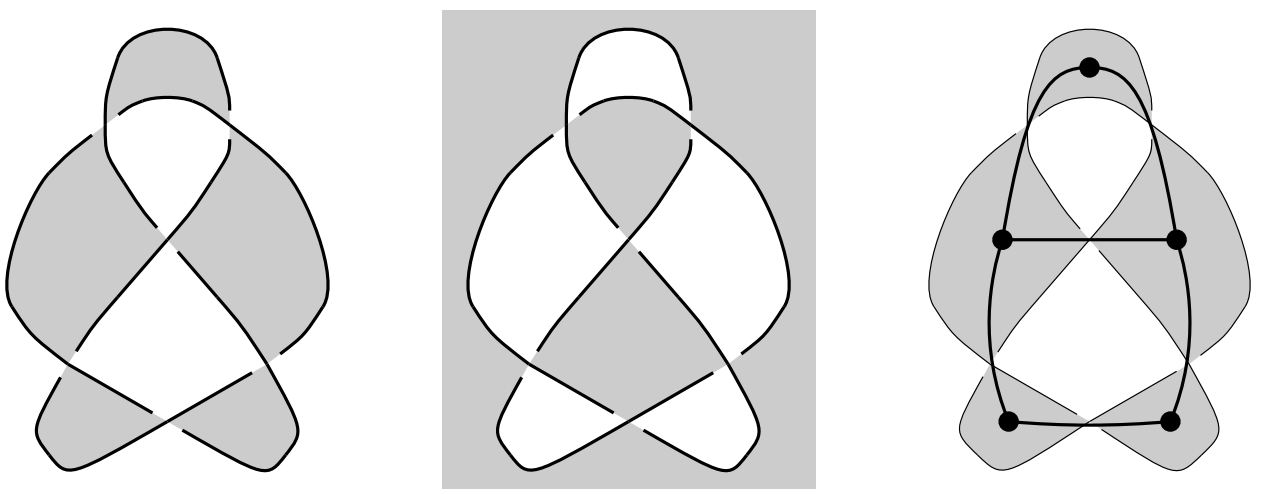

Figure 2. Left and middle: Two checkerboard colorings. Right: checkerboard graph for the first coloring.

In accordance with the ambiguity of $\Gamma(D)$ up to duality, most of the graph properties we will deal with below will be symmetric with respect to duality. We can use this to simplify our arguments at some points. Also, the operations in (3) correspond to contraction and deletion of an edge in the checkerboard graph.

Lemma 3.13. The property that $G=\Gamma(D)$ is 2-connected and (dually) loop-free is equivalent to $D$ having no nugatory crossings.

The calculation of the determinant using monocyclic states can be reformulated even more naturally in terms of graphs. This principle, which we will exploit decisively later, states:

Lemma 3.14. If $D$ is an alternating diagram of a link $L$, and $G=\Gamma(D)$ its checkerboard graph, then $\operatorname{det} L=s(G)$.

Proof. There is a bijection between monocyclic states of $D$ and spanning trees $T$ of $\Gamma(D)$, given by
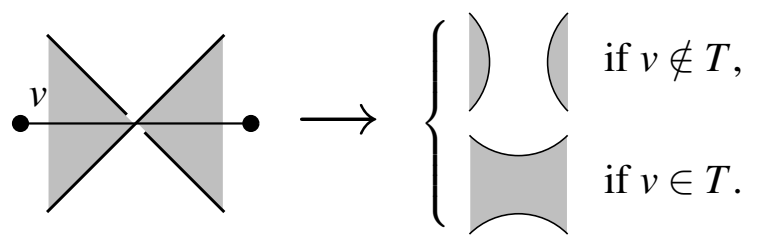

This lemma is well known, and one of its first applications goes probably back to Crowell [1959]. The novel(ty and) additional difficulty below will be to control the extent of the simplification under edge contraction and deletion not only in the checkerboard graph itself but also simultaneously in its planar dual. Lemma 3.14 is also a consequence of Kauffman's state models for the Jones polynomial [Kauffman 1987] and Alexander polynomial [Kauffman 1983], and was discussed extensively in [Stoimenow 2005; Murasugi and Stoimenow 2003]. In this paper, 
we mostly use the language of the latter reference. Our $s(G)$ is written there as $\Delta_{G}(1)$, and in [Stoimenow 2005] as $t(G)$ (a notation which will be used with a different meaning later in this paper).

Twist number of graphs. The Lackenby-Agol-Thurston inequalities are essential in establishing a link between graph theory and the volume. Since, in applying Theorem 2.9, we want to pass from an alternating diagram $D$ to its checkerboard graph $G$, it is useful to remark how twist equivalence translates from $D$ to $G$. The aim of the next definition is to establish this translation. To describe it more precisely, we use some of the above introduced terminology on graphs. Later we need a few more remarks and notions for the analysis of this relation.

Definition 3.15. Let $G$ be a planar 2-connected graph with no loop edges. We call two (simple) edges $e$ and $f$ of $G$ twist equivalent if

(i) $e$ and $f$ are parallel, or

(ii) $\{e, f\}$ is a 2 -cut of $G$.

The twist number $t(G)$ of $G$ is the number of twist equivalence classes of its edges.

Remark 3.16. For $e \neq f$ only one of the alternatives (i) and (ii) is possible, except if $e$ and $f$ are the two edges of $\bigcirc$, occurring as a join factor of $G$.

Lemma 3.17. If $D$ is a link diagram and $G=\Gamma(D)$ its checkerboard graph, then $t(D)=t(G)$.

Proof. This is more or less by definition of $t(G)$. Look at the diagram on the left of (5). It is easy to see that $p$ and $q$ are in the boundary of a common pair of regions (below and above the crossings in the diagram of (5)). Depending on whether these regions are colored black or white in the checkerboard coloring, we obtain the two alternatives in Definition 3.15.

Lemma 3.18. Let $G$ be a planar graph, and $G^{*}$ its dual. Then $s(G)=s\left(G^{*}\right)$, and if $G$ is loop-free and 2-connected, $t(G)=t\left(G^{*}\right)$.

Proof. The coincidence of $s$ already follows from Lemma 3.14, the ambiguity of $\Gamma(D)$ up to duality, and the fact that the determinant is a link invariant. However, there is a much more appropriate way to see this equality. It is a natural bijection between spanning trees of $G$ and $G^{*}$, which is described as follows. Let $T$ be a spanning tree of $G$. To construct the corresponding spanning tree $T^{*}$ of $G^{*}$, include an edge $e^{*}$ of $G^{*}$ into $T^{*}$, if and only if $e$ is not contained in $T$.

As for twist equivalence, note that duality precisely interchanges the conditions (i) and (ii) in Definition 3.15. That is, two edges $e$ and $f$ in $G$ satisfy (i) (respectively (ii)) if and only if $e^{*}$ and $f^{*}$ satisfy (ii) (respectively (i)) in $G^{*}$. 
Lemma 3.19. The number of spanning trees is multiplicative under join, $s\left(G_{1} *\right.$ $\left.G_{2}\right)=s\left(G_{1}\right) \cdot s\left(G_{2}\right)$. The twist number is additive under join of 2-connected loopfree graphs, $t\left(G_{1} * G_{2}\right)=t\left(G_{1}\right)+t\left(G_{2}\right)$.

Proof. For the first claim, note that $T$ is a spanning tree of $G_{1} * G_{2}$, if and only if $T \cap G_{i}$ is a spanning tree of $G_{i}(i=1,2)$.

For the second claim, observe that if two edges are parallel in $G_{1} * G_{2}$, they must be parallel in one of $G_{1}$ or $G_{2}$. If two edges $e_{1,2}$ in $G_{1} * G_{2}$ form a 2-cut, and belong both to one of $G_{1}$ or $G_{2}$, they form a 2-cut in that graph too. If $e_{1}$ is in $G_{1}$ and $e_{2}$ is in $G_{2}$, then they form isthmuses therein, which we excluded. The exclusion of loop edges is needed for "dual" reasons.

Definition 3.20. For a graph $G$ and an edge $e \in E(G)$, we write $G / e$ for the contraction of $e$ in $G$, followed by the deletion of loop edges. Similarly we write $G \backslash e$ for the graph obtained from $G$ by deletion of $e$, and subsequent contraction of all resulting isthmuses:

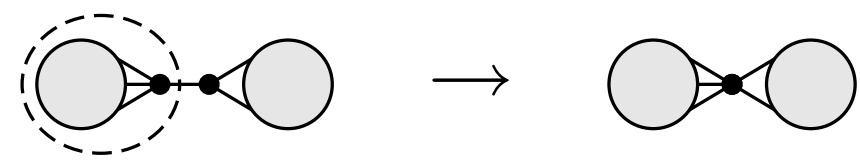

Note again that these operations as dual to each other; $(G / e)^{*}=G^{*} \backslash e^{*}$.

Lemma 3.21. $s(G)=s(G / e)+s(G \backslash e)$.

Proof. Each spanning tree $T$ of $G$ either contains $e$ or it does not. Exactly in former case it descends to a spanning tree $G / e$, and exactly in latter case to one of $G \backslash e$. Contracting isthmuses and deleting loop edges clearly does not affect the number of spanning trees.

Next we consider the behavior of the twist number under deletion and contraction. Here particular attention must be paid to cycles and cuts of length 3 .

Definition 3.22. Let $G$ be a graph and $a$ be an edge of $G$. We call length-3 cycles in $G$ triangles, and denote them by $\triangle$. Abbreviate by ' $a \in n \triangle$ ' the property ' $a$ is contained in (at least) $n$ triangles', and write ' $a \in \triangle^{\prime}$ ' for ' $a \in 1 \triangle$ '. The expression ' $(x, a) \notin \triangle^{\prime}$ ' means that $x$ and $a$ are not both contained in a common triangle etc. We write $\triangle(a)$, the triangle number of $a$, for the number of $\triangle \ni a$. (So for example $a \in 2 \triangle$ if and only if $\Delta(a) \geq 2$.) Similarly we write $c(a)$ for the cut number of $a$, the number of different 3-cuts containing $a$.

Lemma 3.23. If $G$ is 2-connected and loop-free, so are $G \backslash e$ and $G / e$. Moreover, $t(G / e) \geq t(G)-1-\triangle(e)$, and $t(G \backslash e) \geq t(G)-1-c(e)$.

Proof. The first property is clear for loop edges by definition, hence for isthmuses by duality. As for the second, the two inequalities are also dual to each other, so 
we just argue for the first. Contracting an edge does not create any 2-cut; if two edges $f, g \neq e$ form a 2-cut in $G / e$, then so they do in $G$. Contraction may, though, create parallel edges, which happens exactly in $\Delta \ni e$. For each such $\Delta$, the classes of the other two edges are combined into a single class in $G / e$. Additionally we will lose the twist equivalence class of $e$, except possibly when $e$ is in a 2-cut.

\section{Properties of the volume and relation to main results}

Dunfield's experimental observations [2000] result from evaluating the knot tables in Knotscape [Hoste and Thistlethwaite 1999]. This evaluation led to evidence that for alternating links $L$, we have relations of the type

$$
\begin{aligned}
& \operatorname{det} L \approx e^{a \operatorname{vol} L+b}, \text { or } \\
& \operatorname{det} L \approx c(L)^{a \operatorname{vol} L+b}
\end{aligned}
$$

for some numbers $a>0$ and $b$. (Dunfield refers to the degree of the Jones polynomial, but for alternating links this degree was known to be equal to $c(L)$ from [Kauffman 1987; Murasugi 1987; Thistlethwaite 1987].)

If one, however, likes to turn (7) and (8) into rigorous statements valid for all alternating links (and not just for some, even if "generic", subclass), one must make sure to avoid misleading conclusions from the experimental evidence. This requires to take into account some peculiar special cases.

For example, it is easy to construct alternating knots with bounded volume but det $\rightarrow \infty$ (see [Brittenham 1998]), so that an inequality $\leq$ in (7) instead of $\approx$ is impossible. Similarly it is easy to see that an inequality $\geq$ in (8) is impossible. Take for any $a, b$ an alternating knot of sufficiently large volume (which exists for example from the argument of [Lackenby 2004]). Then apply $\bar{t}_{2}^{\prime}$ twists of [Stoimenow 2001] successively at one and the same crossing. Under these moves, the determinant grows linearly, while the volume remains bounded. (In fact it converges to the volume of a certain link by W. Thurston's hyperbolic surgery theorem, as explained in [Brittenham 1998] and [Lackenby 2004].) This in fact shows also that the inequality $\geq$ in (7), stated in Theorem 1.1, is qualitatively close to the best possible, up to at most a linear factor in $c(L)$. That is, for any $a>1$ and any continuous function $f: \mathbb{R}_{+} \rightarrow \mathbb{R}_{+}$, the inequality

$$
\operatorname{det} L>c(L)^{a} \cdot f(\operatorname{vol} L)
$$

cannot hold in general.

Contrarily, the right-hand side of (8) can be made valid (for proper $a$ and $b$ ) as an upper bound on the determinant. As I was informed after my initial writing, such a bound was obtained in unpublished work of Chris Leininger and Ilya Kofman, 
who proved

$$
\operatorname{det} L \leq c(L)^{\operatorname{vol}(L) / V_{0}+2} .
$$

(They have apparently also obtained a special case of Theorem 1.1, with a weaker inequality; compare Proposition 6.3.) In Theorem 1.2 we claimed that we can qualitatively improve such an inequality, by putting vol $L$ into a denominator of the base. We can make the bound more explicit, thus obtaining an improvement of the inequality of Kofman and Leininger.

Theorem 4.1. For any alternating hyperbolic link $L$, we have (with the constants $V_{0}$ and $V_{8}$ of Theorem 2.9)

$$
\operatorname{det} L \leq\left(1+\frac{c(L)}{\max \left(2,1+\frac{\operatorname{vol} L}{10 V_{0}}\right)}\right)^{\frac{\mathrm{vol} L}{V_{8}}+2} .
$$

For Theorem 4.1, we will use some arguments from standard Kauffman bracket skein module theory. (Since they have been repeated many times, we will try to be short; see [Stoimenow 1999; 2005] for more explanations.)

Proof of Theorem 4.1. Let $D$ be a prime alternating diagram of $L$. Without loss of generality, assume all twist equivalent crossings are strongly twist equivalent (in the sense described in Theorem 2.9). Let $t=t(D)$ and $n_{1}, \ldots, n_{t}$, with $\sum_{i=1}^{t} n_{i}=c(D)$, be the cardinalities of the twist equivalence classes of $D$. Each twist equivalence class forms a tangle $T_{i}$. For each $i$, it is an easy exercise to see that exactly $1+n_{i}$ choices of splicings of $T_{i}$ result in two $\operatorname{arcs}\left(n_{i}\right.$ times with one connectivity and once with the other one), and all others have additional circles. In particular, at most $1+n_{i}$ choices of splicings for $T_{i}$ can produce a monocyclic state. So we have

$$
\operatorname{det} D \leq \prod_{i=1}^{t(D)}\left(1+n_{i}\right)
$$

By a computation, if we regard the $n_{i}$ as real variables, then for keeping fixed their sum $c(D)$, the highest possible value on the right-hand side is attained if all the $n_{i}$ are equal to $c(D) / t(D)$. Then use the inequality $t(D) \geq 2$ (valid since $L$ is hyperbolic) and Theorem 2.9.

Remark 4.2. It is easy to construct diagrams $D$ where the splicings of $T_{i}$ of the connectivities occurring $n_{i}$ times join to a monocyclic state, and so

$$
\operatorname{det} D \geq \prod_{i=1}^{t(D)} n_{i}
$$


Therefore, an improvement of Theorem 4.1 beyond removing the additive 1 in the base of the right-hand side of (9) is possible (with this type of argument), only if the inequalities of Theorem 2.9 are improved for such examples.

Proof of Theorem 1.2. We estimate base and exponent on the right of (9) separately. First consider the base. We would like to choose $C_{1}$ so that

$$
\frac{C_{1}}{\operatorname{vol} L} \geq \frac{1}{c(L)}+\frac{1}{\max \left(2,1+\frac{\operatorname{vol} L}{10 V_{0}}\right)} .
$$

Using the inequality vol $L \leq 4 V_{0} c(L)$, explained in [Brittenham 1998; Lackenby 2004], we see that (10) would follow from

which is equivalent to

$$
\frac{C_{1}}{\operatorname{vol} L} \geq \frac{4 V_{0}}{\operatorname{vol} L}+\frac{1}{1+\frac{\operatorname{vol} L}{10 V_{0}}},
$$

$$
\frac{C_{1}-4 V_{0}}{\operatorname{vol} L} \geq \frac{10 V_{0}}{10 V_{0}+\operatorname{vol} L} .
$$

So we see that we can choose $C_{1}=14 V_{0}$.

It remains to estimate the exponent on the right of (9) (from above; keeping in mind that the base in (2) is bigger than 1, as already the one in (9) is). We use the minimality of $\operatorname{vol}\left(4_{1}\right)=2 V_{0}$ among all hyperbolic link volumes [Cao and Meyerhoff 2001], that is, vol $L \geq 2 V_{0}$. Then we conclude that with $C_{2}=1 / V_{0}+$ $1 / V_{8} \approx 1.53115$ we can achieve the stated inequality.

Our main contribution to the proof of the first main result is

Theorem 4.3. For every nontrivial planar 2-connected graph $G$ with no loop edges,

$$
s(G) \geq 2 \cdot \gamma^{t(G)-1},
$$

where $\gamma \approx 1.4253$ is the inverse of the (unique) real positive root of $x^{5}+2 x^{4}+$ $x^{3}-1=0$, given by $\approx 0.701607$.

This easily implies Theorem 1.1.

Proof of Theorem 1.1. If $G$ is the checkerboard graph of an alternating diagram $D$ of $L$, then by Lemma 3.14, $\operatorname{det} L=s(G)$ and by Lemma 3.17, $t(L)=t(D)=t(G)$. By the left (Agol-Thurston) inequality in Theorem 2.9, we have

$$
\operatorname{vol} L \leq 10 V_{0}(t(D)-1)=10 V_{0}(t(G)-1) \leq 10 V_{0} \log _{\gamma} \frac{s(G)}{2},
$$

hence

$$
\operatorname{det} L=s(G) \geq 2 \cdot \gamma^{(\operatorname{vol} L) /\left(10 V_{0}\right)},
$$

and $\gamma^{1 /\left(10 V_{0}\right)} \approx 1.03553$. 


\section{Proof of the spanning tree-twist number inequality}

Induction for nonsimple, 2-connected and decomposable graphs. The proof of Theorem 4.3 underlying Theorem 1.1 is more substantial, and will occupy a significant part of the paper. Let us for later use sort out a few simple cases first, in particular the graphs $G$ with $t(G)=1$, namely those of types

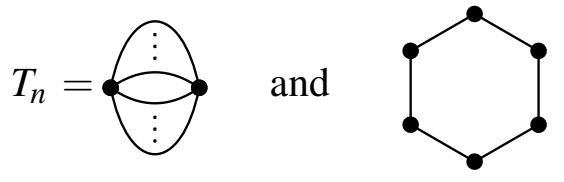

(two-vertex graph and chain).

Lemma 5.1. If $G$ is a join of loop-free 2-connected join factors $G_{i}$ such that every $G_{i}$ has at most 5 edges or $t\left(G_{i}\right)=1$, then $s(G) \geq 2^{t(G)}$.

Proof. It is direct to check that $s\left(G_{i}\right) \geq 2^{t\left(G_{i}\right)}$ for each of the join factors $G_{i}$ of $G$. The inequality for $G$ then follows from Lemmas 3.17 and 3.19, the multiplicativity of $s(G)$ and additivity of $t(G)$ under join.

Proof of Theorem 4.3. We proceed by induction on the number $e(G)$ of edges of $G$. With Lemma 5.1 we can assume that $t(G) \geq 2$ (that is, $G$ is not of the forms in $(11))$ and $e(G) \geq 6$.

In the following, $a$ will be an edge in $G$. Excluding $G$ from being in (11) means that both $G / a$ and $G \backslash a$ (see Definition 3.20) are nontrivial (that is, have $t>0$ ), so that induction applies on both. The aim will be now to choose $a$ so that both $t(G / a)$ and $t(G \backslash a)$ can be controlled from below.

The choice of the constant $\gamma$ will become clear throughout the course of the proof.

We make now a case distinction (exhaustive, but not necessarily exclusive).

Case A. If $G$ has a cut vertex (that is, is decomposable), then we are done inductively by the multiplicativity of $s(G)$ and additivity of $t(G)$ under join (stated in Lemma 3.19), and Lemma 3.6. So we may assume from now on, that $G$ has no cut vertex.

Case B. Assume $G$ has a multiple edge, and let $a$ be one edge of it:

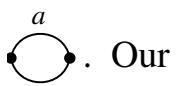
strategy will be to use Lemmas 3.21 and 3.23, and control $c(a)$ and $\triangle(a)$ from above.

Note that (see the proof of Lemma 3.23) the deletion of an edge never changes the number of equivalence classes under the relation given by alternative (ii) in Definition 3.15, while the same holds for contraction of an edge and the alternative (i). Moreover, different twist equivalent edges with property (i) and (ii) are disjoint by Remark 3.16 . 
Since $a$ has a nontrivial twist equivalent edge under property (i), deleting $a$ creates no 1-cut edges (by Remark 3.16). Also, $a$ has a parallel edge, and so the twist equivalence class of $a$ remains in $G \backslash a$ (unless $G$ has $\bigcirc$ as a join factor, which we excluded). Thus if deleting $a$ creates no new 2-cut (that is, $c(a)=0$ ), then $t(G \backslash a)=t(G)$, and induction applies (using Lemma 3.21 and $s(G / a) \geq 0$ ).

So we assume, for the rest of case $\mathrm{B}$, that $c(a)>0$. Then we have (up to a change of the $\infty$-region)

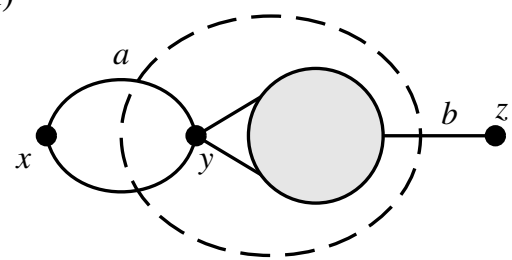

Here $x$ and $z$ may be connected to other vertices outside of the dotted line. Note also that $a$ is not in a (multiple) edge of multiplicity $\geq 3$, because otherwise the only option of having $a$ in a 3 -cut is

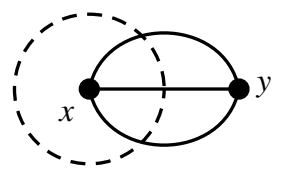

and $e(G)=3$, or one of $x$ or $y$ is a cut vertex, both of which we excluded. Also note that there may be several edges $b$ of the kind shown in (12), but any two of them form a 2-cut.

The reader should now recall the terminology in Definition 3.22 and Lemma 3.23 .

Case B1. Now assume that in a graph $G$ as in (12) we have $a \in \Delta$. Then we have something of the type shown in (13). In this case every $\triangle \ni a$ consists of $a, y z$ and (one of) the (possibly multiple) edge between $x$ and $z$.
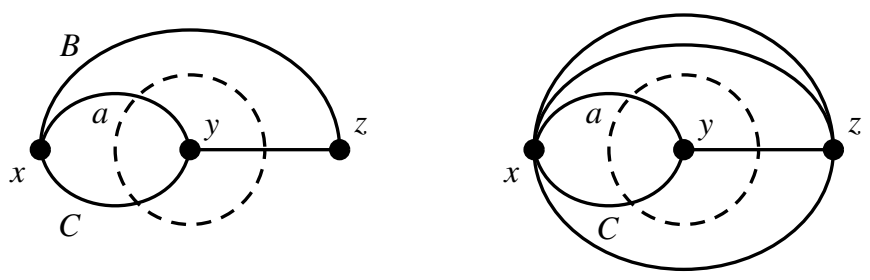

Now consider $G / a$. Then $t(G / a) \geq t(G)-2$, since contracting $a$ we lose its twist equivalence class of edges, and at most two other twist equivalence classes unify to one class under the contraction.

Let $C$ be the region opposite (see Definition 3.8) at the parallel edge $x y$ to $a$ to the bigon region enclosed by $x y$ and $a$. If $x z$ is simple, let $B$ be the region opposite at $x z$ to the $\triangle \ni a$. If $x z$ is multiple, then the specification of $B$ is easily refined, but in fact irrelevant. 
Since $G$ has more than the 4 edges drawn in (13), and $x, y$ and $z$ are not cut vertices, if $x z$ is simple, regions $B$ and $C$ are different, that is, they are separated by edges incident to $x$ and $z$ but not drawn in (12) and (13). If $a$ had now a second 3 -cut, different from the dotted line in (13), the edge $b$ in (12) would not be unique, and so $y z$ in (13) would have a 2-cut. But such a 2-cut would pass through an edge between $x$ and $z$, and thus $x z$ must be simple and $B=C$, which we excluded.

So the dotted line in (13) is the loop of the only 3-cut in which $a$ participates. Then $t(G \backslash a) \geq t(G)-1$, since one identification of twist equivalence classes occurs when deleting $a$, but, as noted, with $a$ being part of a double edge the twist equivalence class of $a$ remains in $G \backslash a$. Then from Lemma 3.21 we have by induction

$$
\begin{aligned}
s(G) & =s(G \backslash a)+s(G / a) \geq 2\left(\gamma^{t(G \backslash a)-1}+\gamma^{t(G / a)-1}\right) \\
& \geq 2\left(\gamma^{t(G)-2}+\gamma^{t(G)-3}\right)=2 \gamma^{t(G)-1}\left(\gamma^{-1}+\gamma^{-2}\right) \geq 2 \gamma^{t(G)-1} .
\end{aligned}
$$

This last inequality follows from the fact that $\gamma \approx 1.4253$, and so $\gamma^{-1}+\gamma^{-2}>1$. (It would suffice here to use the inverse of the positive root of $x+x^{2}-1$.)

Case B2. If $a \notin \triangle$, then we may have several possible $b$ in (12) with which $a$ forms a 3-cut. But we noted that they are all twist equivalent, so still $t(G \backslash a) \geq t(G)-1$, while $a \notin \triangle$ implies also that $t(G / a) \geq t(G)-1$, and thus the estimate (14) applies again.

Case $C$.

If $G$ has a 2-cut, then argue using case B and duality (Lemma 3.18).

\section{Simple 3-connected graphs: properties of 3-cut circuits.}

Case $D$. Thus we can assume now that $G$ has no 2-cut and no double edges (that is, is simple and 3-connected). Then the link diagram $D$ (for which $G=\Gamma(D)$ ) is a polyhedral diagram in the sense of [Conway 1970]. The simplest such diagram has 6 crossings, so we can assume that $t(G)=c(D) \geq 6$.

Now, for each 3-cut we draw a 3-cut circuit in the plane. (We consider these circuits up to homotopy, which does not change the three edges intersected, and does not change the number of intersections with each edge.) We exhibit now in several claims some properties of this collection of circuits.

Claim 5.2. No 3-cut circuit intersects the same edge twice:

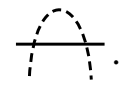

Claim 5.3. We can homotope all 3-cut circuits so that no two of them intersect.

Proof. Assume the contrary, and consider two intersecting 3-cut circuits $a$ and $b$, after homotoping all 3-cut circuits so that they have the minimal possible total number of intersections. We derive a contradiction by showing that one can further 
minimize this number. For this purpose, we define two simple homotopies of a pair of circuits $x$ and $y$, shown in Figure 3, pushing off within a region (a) and pushing off along an edge (b). It is in fact easy to see that these types of homotopies can (and are understood to) be chosen so that the total number of intersections of $x$ and $y$ with other circuits is not augmented.

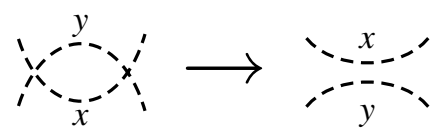

(a)

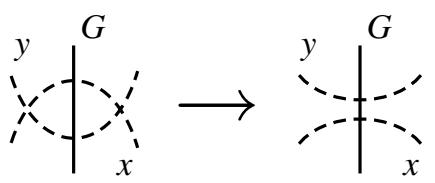

(b)

Figure 3. Cut circuit homotopies that reduce the number of intersections: pushing off within a region (a) and pushing off along an edge (b). In both cases, we assume no part of $G$ - other than the drawn edge fragment in (b) - enters the bigon present at the beginning of the move.

If $a$ and $b$ intersect in only one region of $G$, then they can be pushed off each other within that region. So assume $a$ and $b$ intersect in at least two regions of $G$, say $A$ and $B$.

Consider $G^{*}$. Since $G$ is simple and 3-connected, so is $G^{*}$. Any 3-cut circuit $x$ of $G$ corresponds in $G^{*}$ (in the sense of Definition 3.9) to a cycle $x^{*}$ of length 3 , that is, a triangle of $G^{*}$. Thus $a^{*}$ and $b^{*}$ are two triangles in $G^{*}$ which share a pair of common vertices $A^{*}$ and $B^{*}$. Now by simplicity of $G^{*}$, any two distinct triangles in it which meet in two vertices, must share the unique edge between these vertices. Thus $a$ and $b$ pass between $A$ and $B$ through the same edge of $G$. Then it is clear that $a$ and $b$ can be pushed off each other along this edge.

We assume from now on that all 3-cut circuits are homotoped so as to be disjoint.

Claim 5.4. No two 3-cuts have a pair of common edges.

Proof. Assume there were such two 3-cuts, and pair of edges $e$ and $f$, and consider the cut circuits. By 3-connectivity of $G$, no edge bounds the same region from both sides, and each pair of edges has at most one common region $R$. For $e$ and $f$ clearly $R$ must exist, and then both circuits must pass (without intersecting, by Claim 5.3) through $R$. By the Jordan curve theorem no circuit can leave $R$ and enter it again (more than once). Thus we have the situation on the left:

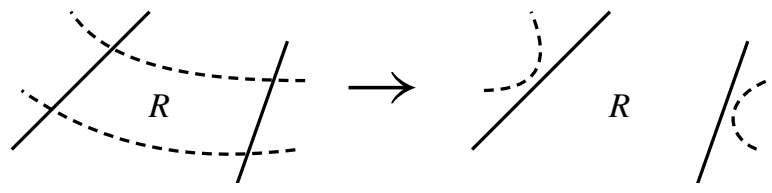


Modifying this to the situation on the right gives a 2-cut circuit (with two different edges intersected, because the original two 3-cuts were different). This is a contradiction.

The next result is a direct consequence of Claim 5.4.

Claim 5.5. If $E$ is a triangle (that is, a cycle of length 3; not necessarily the boundary of a face), then all three edges in E have cut number at most two.

Claim 5.6. If $E$ is $a \leq 5$-gonal face (that is, a face bounded by at most 5 edges), then for each pair $e$ and $f$ of neighbored edges in $\partial E$ there exists an edge a $\in$ $\partial E \backslash\{e, f\}$ with $c(a) \leq 2$. (Neighbored edges means edges incident to a common vertex, which is a corner of $E$.)

Proof. If we assume that $c(a) \geq 3$ for all $a \notin\{e, f\}$, it is an easy exercise to check that one cannot install the pieces of the arcs of the 3-cut circuits within $E$ so that the conditions of the first 3 claims are satisfied simultaneously.

Claim 5.7. Assume $l$ is a simple closed curve intersecting $n$ edges of $G$ transversely, and (among these edges) $a$ exactly once. Then $n>\Delta(a)$.

Proof. Let $B$ and $C$ be the two regions bounded by $a$, and $l$ be oriented so as to pass from $B$ to $C$ through $a$. This passage changes the inside/outside position of the curve $l$ with respect to any of the triangles containing $a$. Thus, in order to return from $C$ to $B$, the closed curve $l$ must intersect at least one (further) edge for each triangle containing $a$, and these edges are all distinct by Claim 5.4.

Completing the induction: finding a good edge. With the above claims prepared, we continue with case D. Let $G$ be simple and 3-connected. It is well known, that in every planar embedding of $G$ there is a $\leq 5$-gonal face $E$. Let $a$ be an edge in its boundary $\partial E$.

If we now choose $a \in \partial E$ to have at most two 3-cut circuits intersecting it, by Lemma 3.23, we have $t(G \backslash a) \geq t(G)-3$, since deleting $a$ we lose its twist equivalence class, and at most two pairs of other twist equivalence classes identify. It remains to refine the choice of $a$ so as to control also $t(G / a)$. To this vein, we must count again the triangles containing $a$. Since we assumed that $G$ has no double edge, two such triangles have only $a$ as a common edge.

We claim that one can find an edge $a$ so that

(i) $\triangle(a) \leq 2$ and $c(a) \leq 2$, but

(ii) unless $G$ is the graph in (17) (see page 443), it is not the case that $\triangle(a)=$ $c(a)=2$.

We describe an algorithm to find some edge $a$ satisfying (i). (Here the choice of the $\infty$ region is kept fixed!) Condition (ii) will be dealt with subsequently. 
(1) Start with some edge $a \in \partial E$ with $c(a) \leq 2$. Such an edge exists by Claim 5.6.

(2) If $a \in 3 \triangle$, there is always a pair among these three $\Delta$ such that one triangle encloses the other:

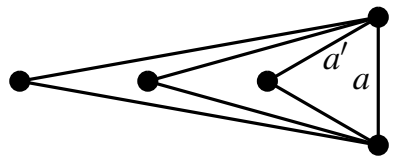

or

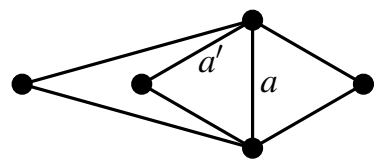

(There may be further edges in the interior of these triangles.) Note in particular that the valence of the two vertices connected by $a$ is higher than the number of different $\triangle \ni a$.

(3) Take $a^{\prime} \neq a$ in the boundary of an innermost triangle from the ones in the figure immediately above. Set $a:=a^{\prime}$ and go to step (2).

Since this iteration augments the number of $\triangle$ enclosing $a$ (and for any edge of $G$ there are only finitely many such triangles), at some point the test in (2) fails, and $a$ is in at most 2 triangles. Since $a \in \Delta$, we have $c(a) \leq 2$ by Claim 5.5.

This algorithm gives an edge $a$ which is in at most two triangles, and at most two cuts. We must show now that we can avoid $a$ being in exactly two triangles and exactly two cuts.

Assume that any $a$ with $c(a) \leq 2$ and $\triangle(a) \leq 2$ has $c(a)=\triangle(a)=2$. Let $E_{1}$, $E_{2}$ be the $2 \triangle \ni a$.

Now $a$ is in two 3-cuts, and by Claim 5.4 each cut must pass through one other edge of each of the $\Delta \ni a$. By Claim 5.7, then there are at most two triangles containing $a$, and both cut circuits do not pass through any further edges. Thus the vertices $v$ and $w$ that $a$ connects are trivalent, and the cut circuits are going around them (that is, their interior contains this one single vertex):

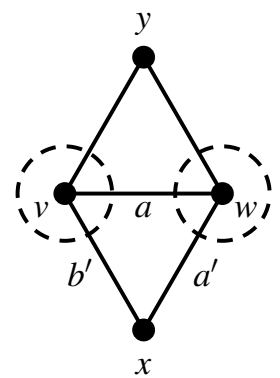

Then $v, w$ cannot be incident to edges in the interior of $E_{1}$. Now $E_{1}$ is a triangle, and its other vertex, different from $v, w$, is not a cut vertex (as $G$ has no cut vertex by assumption). So $E_{1}$ must have empty interior, that is, be a triangular face. The same assertion similarly follows for $E_{2}$. 
(Actually, one may need to replace "interior" by "exterior" in this argument, for one of $E_{1}$ or $E_{2}$; it depends on the choice of the $\infty$ region. We used "interior" to keep the wording simple. Alternatively, one could avoid considering the exterior, if one continues the iteration in part (2) of the algorithm above even if $\triangle(a)=2$, as long as one $\triangle \ni a$ encloses the other.)

Since all the 4 edges in $\partial E_{1} \cup \partial E_{2} \backslash\{a\}$ are incident to a trivalent vertex, by Claim 5.7 they cannot belong to more than two triangles. But since they do belong to a triangle, by Claim 5.5 they cannot have cut number $>2$. Thus assume all they belong to (exactly) two triangles and two 3-cuts - otherwise we can choose $a$ to be one of them, and we are done.

Then consider $a^{\prime}$ in (16) instead of $a$. The only way in which $a^{\prime}$ can have a second 3-cut $X$ conforming to Claims 5.2, 5.3 and 5.4 is if $X$ goes through $a^{\prime}$ and $b^{\prime}$. Then the same argument as for $a$ shows that $a^{\prime} \in 2 \triangle$ only if $x$ is trivalent and connected to a vertex, which is different from $v$ and connected to $w$. The only such vertex is $y$. Thus we have

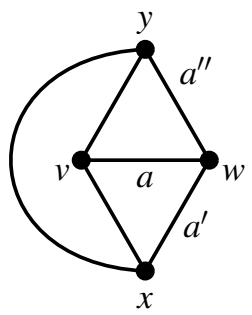

Now, arguing for $a^{\prime \prime}$ the same way as with $a$, we see that $y$ is also trivalent, and that hence $G$ has no more edges than those 6 drawn in (17). This graph $G$ has 16 spanning trees and $t(G)=6$, and the inequality in the theorem is directly verified. Otherwise one of the 4 edges in $\partial E_{1} \cup \partial E_{2} \backslash\{a\}$ has at most one cut, and we found the edge we sought.

Having found this edge $a$, we reach one of two cases:

$$
\begin{aligned}
& t(G \backslash a) \geq t(G)-2 \text { and } t(G / a) \geq t(G)-3, \text { or } \\
& t(G \backslash a) \geq t(G)-3 \text { and } t(G / a) \geq t(G)-2 .
\end{aligned}
$$

This way we could already conclude the theorem with a base of the exponential being the inverse of the real positive root of $x^{3}+x^{2}-1$. However, we can do a bit better in applying induction. (Recall the remarks on the polynomials we made at the beginning of the proof.)

Case D1. If none of $c(a)$ and $\triangle(a)$ is zero, then observe that both $G / a$ and $G \backslash a$ contain a multiple edge respectively a 2-cut. Assume that $t(G)-t(G \backslash a) \leq 3$ and $t(G)-t(G / a) \leq 2$; the reverse case is symmetric with respect to the argument that follows (only the estimates in (19) and (20) would interchange). 
Case Dla. If $G / a$ has a join factor $G^{\prime}$ with $e\left(G^{\prime}\right) \leq 5$ or $t\left(G^{\prime}\right)=1$, let $G^{\prime \prime}$ with $G / a=G^{\prime} * G^{\prime \prime}$ be the (join of the) other factor(s). Then apply Lemma 5.1 on $G^{\prime}$ and induction on $G^{\prime \prime}$. We obtain, using also Lemma 3.19,

$$
\begin{aligned}
s(G / a) & =s\left(G^{\prime}\right) \cdot s\left(G^{\prime \prime}\right) \geq 2^{t\left(G^{\prime}\right)} \cdot 2 \gamma^{t\left(G^{\prime \prime}\right)-1}=2 \cdot 2^{t\left(G^{\prime}\right)-1} \cdot 2 \gamma^{t\left(G^{\prime \prime}\right)-1} \\
& \geq 4 \cdot \gamma^{t\left(G^{\prime}\right)+t\left(G^{\prime \prime}\right)-2}=4 \gamma^{t(G / a)-2} \geq 4 \gamma^{t(G)-4} .
\end{aligned}
$$

We will see that this estimate subsumes the one in the next case.

Case $D 1 b$. If $G / a$ has no such join factor, the multiple edge respectively 2-cut is contained in a join factor $G^{\prime}$ of $G / a$ which is recursively subjectable to cases B or C. This means that we can find an edge $a^{\prime}$ in $G^{\prime}$, and hence $G / a$, such that the graphs $G_{c c}=(G / a) / a^{\prime}$ and $G_{c d}=(G / a) \backslash a^{\prime}$, obtained by contracting respectively deleting $a^{\prime}$ in $G / a$, satisfy (up to interchange) $t\left(G_{c c}\right) \geq t(G / a)-2 \geq t(G)-4$ and $t\left(G_{c d}\right) \geq t(G / a)-1 \geq t(G)-3$. (Here again Lemma 3.19 is tacitly applied.) Then

$$
s(G / a) \geq 2 \gamma^{t(G)-5}+2 \gamma^{t(G)-4} .
$$

Since (18) is stronger, it is enough to keep working with (19).

Similarly, using graphs $G_{d c}=(G \backslash a) / a^{\prime}, G_{d d}=(G \backslash a) \backslash a^{\prime}$ with $t\left(G_{d c}\right) \geq t(G \backslash$ $a)-2 \geq t(G)-5$ and $t\left(G_{d d}\right) \geq t(G \backslash a)-1 \geq t(G)-4$ (again up to interchange), we obtain for $s(G \backslash a)$ the inequality

$$
s(G \backslash a) \geq 2 \gamma^{t(G)-5}+2 \gamma^{t(G)-6} .
$$

(There is no problem in writing down these quantities because we ensured in the beginning of case D that $t(G) \geq 6$. So none of $G_{c c}, G_{c d}, G_{d c}, G_{d d}$ is the trivial 1-vertex graph.) Using inequalities (19) and (20) and Lemma 3.21, we have

$$
\begin{aligned}
s(G) & =s(G \backslash a)+s(G / a) \\
& \geq 2 \gamma^{t(G)-5}+2 \gamma^{t(G)-4}+2 \gamma^{t(G)-5}+2 \gamma^{t(G)-6} \\
& =2 \gamma^{t(G)-1}\left(\gamma^{-5}+2 \gamma^{-4}+\gamma^{-3}\right)=2 \gamma^{t(G)-1} .
\end{aligned}
$$

This last equality uses the fact that $1 / \gamma$ is a root of $x^{5}+2 x^{4}+x^{3}-1$ (and so explains our choice of $\gamma$ ). Then we obtain the claim.

Case D2. If some of $c(a)$ and $\triangle(a)$ is zero (and the other one is at most two), we obtain the recursive estimate

$$
s(G) \geq 2 \gamma^{t(G)-1}\left(\gamma^{-3}+\gamma^{-1}\right) .
$$

In order the right-hand side to evaluate to at least $2 \gamma^{t(G)-1}$, we need $1 / \gamma$ to be not smaller than the (real positive) root of the polynomial $x^{3}+x-1$. But one indeed 
checks that this polynomial is positive for $x=\gamma^{-1}$. In other words, the $\gamma$ we have chosen so far is smaller than the one we would need for this recursive estimate.

Now the proof of Theorem 4.3 is complete.

\section{Possible improvements, computations and examples}

Let us conclude the topic with some observations on experimental data and partial improvements.

\section{Alternating arborescent links.}

Definition 6.1. The doubling of an edge (installing an edge connecting the same two vertices) is the operation

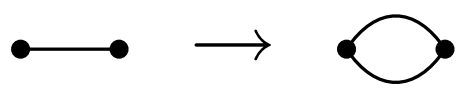

The bisection of an edge (putting a valence- 2 vertex on it) is the operation

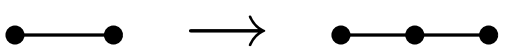

(Other edges may be incident from the left- and rightmost vertices on both sides, and the rest of the graphs on both sides are assumed to be equal.)

For a graph $G^{\prime}$ let $\left\langle G^{\prime}\right\rangle$ be the family of graphs obtained from $G^{\prime}$ by repeated edge bisections and doublings. A graph $G$ is series parallel if $G \in\langle\bullet \bullet\rangle$. We say that $G$ is $n$-series parallel if $G \in\left\langle G^{\prime}\right\rangle$ and $G^{\prime}$ is a simple 3-connected planar graph of $n$ edges.

Series parallel graphs correspond, via the checkerboard construction, to alternating arborescent link diagrams, that is, alternating diagrams with Conway polyhedron $1^{*}$ [1970]. More generally, link diagrams with an $n$-crossing Conway polyhedron come from $n$-series parallel graphs.

For a series parallel graph, one can use the proof of Theorem 4.3 to obtain, more easily, a better inequality. Here $T_{2}$ is the two-vertex graph with a double edge as in (11) (and Remark 3.16), and $F_{i}$ denote the Fibonacci numbers, defined by $F_{1}=1$, $F_{2}=1$ and $F_{n}=F_{n-1}+F_{n-2}$ for $n>2$.

Proposition 6.2. If $G$ is series parallel, or the join of such graphs, and $G \neq$ $T_{2}, T_{2} * T_{2}$, then

$$
s(G) \geq F_{t(G)+3} .
$$

Proof. For a series parallel graph, case 5 in the induction of the proof of Theorem 4.3 never occurs. So we can use the estimate (14), which yields the recursion of the Fibonacci numbers. With that argument completed, now one needs to take care only of graphs $G$, for which $G / a$ or $G \backslash a$ is one of $T_{2}$ or $T_{2} * T_{2}$. The proof shows 
that such graphs $G$ have twist number at most 4, and they can be easily dealt with. (It suffices to check graphs with at most two edges in each twist equivalence class, and so at most 8 edges.)

Note that if $G_{1,2}$ are series parallel, then one can always perform the join $G_{1} * G_{2}$ (by proper choice of join vertices) so that the result is series parallel, but not every possible join will be so.

The inequality (21) can be realized sharply (that is, as equality) taking (checkerboard graphs of) rational link diagrams $(21 \ldots 12)$, so it is optimal. This inequality in particular allows us to improve the base of the exponential in Theorem 1.1 for alternating arborescent links (which seems related to the partial cases obtained also by Kofman and Leininger).

Proposition 6.3. If $L$ is a hyperbolic alternating arborescent link, then $\operatorname{det} L \geq$ $1.4424 \cdot 1.0485^{\mathrm{vol} L}-0.0118$.

Proof. If an alternating link $L$ is arborescent, then an alternating diagram $D$ of $L$ has a Conway polyhedron $1^{*}$ or $6^{*}$; see [Thistlethwaite 1991]. This means that its checkerboard graph $G \in\langle 1\rangle \cup\left\langle G^{\prime}\right\rangle$, where we write 1 for $\bullet$ and $G^{\prime}$ is the graph in (17). For $G \in\langle 1\rangle$, Proposition 6.2 applies directly (the two exceptional $G$ yield nonhyperbolic $L$ ). For $G \in\left\langle G^{\prime}\right\rangle$ the same inductive argument as in the proof of Proposition 6.2 can be used, unless $G=G^{\prime}$, a situation which we examined separately already. In all other cases, the proof of Theorem 4.3 shows that, with an edge $a$ chosen properly, $t(G / a), t(G \backslash a) \geq t(G)-3$, so if $G \in\left\langle G^{\prime}\right\rangle$, then $t(G) \geq 6$, and none of $G / a, G \backslash a$ is $T_{2}$ or $T_{2} * T_{2}$. Moreover, if $G \in\langle 1\rangle \cup\left\langle G^{\prime}\right\rangle$, then the same holds for $G / a$ and $G \backslash a$. By adapting constants to $G^{\prime}$, where $s=16$ and $F_{9}=34$, we have

$$
\operatorname{det} D \geq \frac{8}{17} F_{t(D)+3} \text {. }
$$

Now from (22), using $t(D) \geq 2$, the explicit formula

$$
F_{n}=\frac{1}{\sqrt{5}}\left(\left(\frac{1+\sqrt{5}}{2}\right)^{n}-\left(\frac{1-\sqrt{5}}{2}\right)^{n}\right)
$$

and the Agol-Thurston bound in Theorem 2.9, we find

$$
\begin{aligned}
\operatorname{det} L & \geq \frac{8}{17 \sqrt{5}}\left(\frac{1+\sqrt{5}}{2}\right)^{\frac{\mathrm{vol} L}{10 V_{0}}+4}-\frac{8}{17 \sqrt{5}}\left(\frac{\sqrt{5}-1}{2}\right)^{6} \\
& \approx 1.44247 \cdot 1.04855^{\mathrm{vol} L}-0.01172,
\end{aligned}
$$

which implies the estimate.

Remark 6.4. There is some annoying confusion as to the meaning of "alternating arborescent link". As we explained, there are links which are alternating and 
arborescent, but whose alternating diagrams are not arborescent. (The Borromean rings, whose checkerboard graph is $G^{\prime}$, are the simplest example.) These pathologies are often tacitly ignored by many uncareful authors. If we consider alternatingly arborescent links, that is, links whose alternating diagrams are arborescent, then the treatment of $G^{\prime}$ and the constant $\frac{8}{17}$ in (22) are not needed, and we can slightly improve our estimate.

Experimental and computational remarks. A natural question is to what extent the base in (1) can be improved in general. By refining the argument that led to the occurrence of the polynomial $x^{5}+2 x^{4}+x^{3}-1$, one could try to 'push' the zero below that of $x^{3}+x-1$, and then have a better (and writable in radicals) constant $\gamma$ in Theorem 4.3. Such an improvement seems very burdensome and little efficient, though.

To obtain some idea about the maximum possible, we evaluated (neglecting the unnatural multiplicative factor in (1)) for alternating hyperbolic knots $K$ the invariant $\mu(K):=\sqrt[\operatorname{vol} K]{\operatorname{det} K}$. The smallest value among alternating knots of at most 16 crossings was found attained by $16_{375396}$ with $\mu \approx 1.2507$. In general the minimal $\mu$ decreases with increasing crossing number. It is somewhat surprising that in fact the knots of largest determinant [Stoimenow 2007] have the smallest or second smallest (for 10,11, 16 crossings) $\mu$.

Since the numeric data suggests (as expected) space for improvement, a further question is where such improvement is possible. Theorem 1.1 is a coupling of two independent parts, Theorem 4.3 and Theorem 2.9. As we observed, the base $\gamma \approx 1.42$ in Theorem 4.3 is likely not optimal, but can not be increased beyond $\frac{1}{2}(1+\sqrt{5}) \approx 1.62$. Contrarily, while Agol-Thurston show that their inequality is (asymptotically) sharp in general, in practice it often fails considerably. We examined the inequality

$$
\frac{\operatorname{vol} K}{10 V_{0}(t(K)-1)}<\frac{(\ln \gamma) \cdot(t(K)-1)}{\ln (\operatorname{det} K / 2)},
$$

whose two sides compare the unsharpness of the estimates in Theorems 4.3 and 2.9. For example, among the 6723 hyperbolic alternating knots $K$ of $\leq 13$ crossings, 6273 (or $\approx 93.3 \%$ ) satisfy (23). Such evidence suggests that the loss of quality in the constant in Theorem 1.1 is to a larger extent due to the application of Theorem 2.9, rather than Theorem 4.3. This, however, seems natural, since the volume is more complex to understand than the graphs.

We make a final remark that, apart from estimates, an exact relation between determinant and volume is unlikely to exist. Examples of (even alternating) knots with equal determinant but different volume are easy to find, and, at least including nonalternating knots, there are well known pairs with equal volume but different 


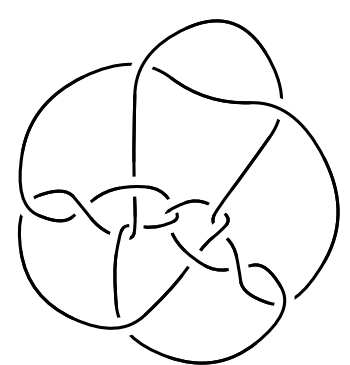

$15_{79500}$

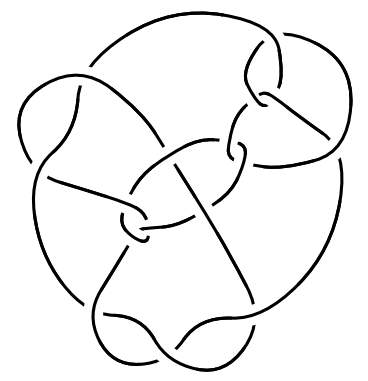

165435

Figure 4. Two alternating knots with (almost?) equal volume but different determinant.

determinant, for instance $9_{42}$ and $10_{132}$. For alternating knots such pairs are not obvious, but one has situations where the volumes are very close to each other.

Example 6.5. The best pair I have found that sufficiently demonstrates the problem is $15_{79500}$ and $16_{5435}$ (see Figure 4). Their polynomials and determinants are quite different, while their volumes differ only by $10^{-10}$, when calculated up to this same precision $\left(\operatorname{vol}\left(15_{79500}\right) \approx 27.3456887210\right.$, and $\left.\operatorname{vol}\left(16_{5435}\right) \approx 27.3456887209\right)$. Since such computational deviations occur even among different diagrams of the same knot, the volumes may even be equal. (Note that, in very contrast to discrete invariants, to decide whether two volumes are equal is not always trivial.)

\section{Acknowledgements}

I would like to thank Nathan Dunfield, Efstratia Kalfagianni, Mikhail Khovanov, Chris Leininger, Kunio Murasugi and Dan Silver for helpful remarks, discussions, and references. The appearance of the paper was most significantly influenced by the comments of the referee, who deserves little short of coauthorship for his or her contribution. This research was partly funded by Postdoc grant P04300 of the Japan Society for the Promotion of Science (JSPS) at the University of Tokyo. I also thank my JSPS host, Prof. T. Kohno, for his support.

\section{References}

[Agol and Thurston 2004] I. Agol and D. Thurston, Appendix to [Lackenby 2004].

[Agol et al. 2007] I. Agol, P. A. Storm, and W. P. Thurston, "Lower bounds on volumes of hyperbolic Haken 3-manifolds", J. Amer. Math. Soc. 20:4 (2007), 1053-1077.

[Alexander 1928] J. W. Alexander, "Topological invariants of knots and links", Trans. Amer. Math. Soc. 30:2 (1928), 275-306. MR 1501429 JFM 54.0603.03

[Brandt et al. 1986] R. D. Brandt, W. B. R. Lickorish, and K. C. Millett, "A polynomial invariant for unoriented knots and links", Invent. Math. 84:3 (1986), 563-573. MR 87m:57003 Zbl 0595.57009 
[Brittenham 1998] M. Brittenham, "Bounding canonical genus bounds volume”, preprint, 1998, available at http://www.math.unl.edu/ mbritten/personal/pprdescr.html.

[Cao and Meyerhoff 2001] C. Cao and G. R. Meyerhoff, "The orientable cusped hyperbolic 3manifolds of minimum volume", Invent. Math. 146:3 (2001), 451-478. MR 2002i:57016 Zbl 1028.57010

[Conway 1970] J. H. Conway, "An enumeration of knots and links, and some of their algebraic properties", pp. 329-358 in Computational Problems in Abstract Algebra (Oxford, 1967), edited by J. Leech, Pergamon, Oxford, 1970. MR 41 \#2661 Zbl 0202.54703

[Crowell 1959] R. H. Crowell, "Nonalternating links", Illinois J. Math. 3 (1959), 101-120. MR 20 \#6105 Zbl 0119.38802

[Dasbach and Hougardy 1996] O. T. Dasbach and S. Hougardy, "A conjecture of Kauffman on amphicheiral alternating knots”, J. Knot Theory Ramifications 5:5 (1996), 629-635. MR 97h:57015 Zbl 0890.57011

[Dasbach and Lin 2007] O. T. Dasbach and X.-S. Lin, "A volume-ish theorem for the Jones polynomial of alternating knots", Pacific J. Math. 231 (2007), 279-291.

[Dunfield 2000] N. Dunfield, "An interesting relationship between the Jones polynomial and hyperbolic volume", web page, 2000, available at http://www.its.caltech.edu/ dunfield/preprints/misc/ dylan.

[Freyd et al. 1985] P. Freyd, D. Yetter, J. Hoste, W. B. R. Lickorish, K. Millett, and A. Ocneanu, "A new polynomial invariant of knots and links", Bull. Amer. Math. Soc. (N.S.) 12:2 (1985), 239-246. MR 86e:57007 Zbl 0572.57002

[González-Acuña and Short 1991] F. González-Acuña and H. Short, "Cyclic branched coverings of knots and homology spheres”, Rev. Mat. Univ. Complut. Madrid 4 (1991), 97-120. MR 93g:57004

[Gordon 1972] C. M. Gordon, "Knots whose branched cyclic coverings have periodic homology", Trans. Amer. Math. Soc. 168 (1972), 357-370. MR 45 \#4394 Zbl 0238.55001

[Gordon and Litherland 1978] C. M. Gordon and R. A. Litherland, "On the signature of a link", Invent. Math. 47:1 (1978), 53-69. MR 58 \#18407 Zbl 0391.57004

[Hartley and Kawauchi 1979] R. Hartley and A. Kawauchi, "Polynomials of amphicheiral knots", Math. Ann. 243:1 (1979), 63-70. MR 81c:57004 Zbl 0394.57009

[Hoste and Thistlethwaite 1999] J. Hoste and M. Thistlethwaite, KnotScape, 1999, available at http:// www.math.utk.edu/ morwen/knotscape.html. Software for knot polynomial calculation and table access.

[Jones 1985] V. F. R. Jones, “A polynomial invariant for knots via von Neumann algebras”, Bull. Amer. Math. Soc. (N.S.) 12:1 (1985), 103-111. MR 86e:57006 Zbl 0564.57006

[Jones 1987] V. F. R. Jones, "Hecke algebra representations of braid groups and link polynomials", Ann. of Math. (2) 126:2 (1987), 335-388. MR 89c:46092 Zbl 0631.57005

[Kalfagianni 2004] E. Kalfagianni, "Alexander polynomial, finite type invariants and volume of hyperbolic knots", Algebr. Geom. Topol. 4 (2004), 1111-1123. MR 2005k:57017 Zbl 1078.57014

[Kauffman 1983] L. H. Kauffman, Formal knot theory, Mathematical Notes 30, Princeton University Press, Princeton, NJ, 1983. MR 85b:57006 Zbl 0537.57002

[Kauffman 1987] L. H. Kauffman, "State models and the Jones polynomial”, Topology 26:3 (1987), 395-407. MR 88f:57006 Zbl 0622.57004

[Kauffman 1990] L. H. Kauffman, “An invariant of regular isotopy”, Trans. Amer. Math. Soc. 318:2 (1990), 417-471. MR 90g:57007 Zbl 0763.57004 
[Khovanov 2000] M. Khovanov, "A categorification of the Jones polynomial”, Duke Math. J. 101:3 (2000), 359-426. MR 2002j:57025 Zbl 0960.57005

[Krebes 1999] D. A. Krebes, "An obstruction to embedding 4-tangles in links", J. Knot Theory Ramifications 8:3 (1999), 321-352. MR 2000h:57012 Zbl 0941.57012

[Lackenby 2004] M. Lackenby, "The volume of hyperbolic alternating link complements", Proc. London Math. Soc. (3) 88:1 (2004), 204-224. MR 2004i:57008 Zbl 1041.57002

[Menasco 1984] W. Menasco, "Closed incompressible surfaces in alternating knot and link complements”, Topology 23:1 (1984), 37-44. MR 86b:57004 Zbl 0525.57003

[Menasco and Thistlethwaite 1991] W. W. Menasco and M. B. Thistlethwaite, "The Tait flyping conjecture”, Bull. Amer. Math. Soc. (N.S.) 25:2 (1991), 403-412. MR 92b:57017 Zbl 0745.57002

[Menasco and Thistlethwaite 1993] W. Menasco and M. Thistlethwaite, "The classification of alternating links", Ann. of Math. (2) 138:1 (1993), 113-171. MR 95g:57015 Zbl 0809.57002

[Murakami and Murakami 2001] H. Murakami and J. Murakami, "The colored Jones polynomials and the simplicial volume of a knot”, Acta Math. 186:1 (2001), 85-104. MR 2002b:57005 Zbl 0983.57009

[Murasugi 1987] K. Murasugi, "Jones polynomials and classical conjectures in knot theory", Topology 26:2 (1987), 187-194. MR 88m:57010 Zbl 0628.57004

[Murasugi and Stoimenow 2003] K. Murasugi and A. Stoimenow, "The Alexander polynomial of planar even valence graphs", Adv. in Appl. Math. 31:2 (2003), 440-462. MR 2004i:05038 Zbl 1029.05039

[Riley 1990] R. Riley, "Growth of order of homology of cyclic branched covers of knots", Bull. London Math. Soc. 22:3 (1990), 287-297. MR 92g:57017 Zbl 0727.57002

[Rolfsen 1976] D. Rolfsen, Knots and links, Mathematics Lecture Series 7, Publish or Perish, Berkeley, 1976. MR 58 \#24236 Zbl 0339.55004

[Silver and Whitten 2005] D. S. Silver and W. Whitten, "Hyperbolic covering knots", Algebr. Geom. Topol. 5 (2005), 1451-1469. MR 2006i:57019 Zbl 1085.57009

[Silver et al. 2006] D. S. Silver, A. Stoimenow, and S. G. Williams, "Euclidean Mahler measure and twisted links”, Algebr. Geom. Topol. 6 (2006), 581-602. MR 2007c:57018 Zbl 1096.57013

[Stoimenow 1999] A. Stoimenow, "The Jones polynomial, genus and weak genus of a knot", Ann. Fac. Sci. Toulouse Math. (6) 8:4 (1999), 677-693. MR 2002a:57024 Zbl 0995.57002

[Stoimenow 2001] A. Stoimenow, "Knots of genus one or on the number of alternating knots of given genus", Proc. Amer. Math. Soc. 129:7 (2001), 2141-2156. MR 2002c:57012 Zbl 0971.57012

[Stoimenow 2003] A. Stoimenow, "Knots of genus two", preprint, 2003. math.GT/0303012

[Stoimenow 2005] A. Stoimenow, "Square numbers, spanning trees and invariants of achiral knots", Comm. Anal. Geom. 13:3 (2005), 591-631. MR 2007b:57025 Zbl 02247291

[Stoimenow 2006] A. Stoimenow, "Coefficients and non-triviality of the Jones polynomial", preprint, 2006. math.GT/0606255

[Stoimenow 2007] A. Stoimenow, "Maximal determinant knots", Tokyo J. Math. 30:1 (2007), 7397. MR 2328056

[Thistlethwaite 1987] M. B. Thistlethwaite, "A spanning tree expansion of the Jones polynomial", Topology 26:3 (1987), 297-309. MR 88h:57007 Zbl 0622.57003

[Thistlethwaite 1988] M. B. Thistlethwaite, "On the Kauffman polynomial of an adequate link", Invent. Math. 93:2 (1988), 285-296. MR 89g:57009 Zbl 0645.57007

[Thistlethwaite 1991] M. B. Thistlethwaite, "On the algebraic part of an alternating link", Pacific J. Math. 151:2 (1991), 317-333. MR 92m:57016 Zbl 0745.57003 
[Wendt 1937] H. Wendt, "Die gordische Auflösung von Knoten”, Math. Z. 42:1 (1937), 680-696. MR 1545700 JFM 0016.42005

Received May 24, 2006. Revised July 29, 2007.

\author{
Alexander Stoimenow \\ RESEARCh InStitute FOR Mathematical SCIENCES \\ KYOTO UNIVERSITY \\ КYOTO 606-8502 \\ JAPAN \\ stoimeno@kurims.kyoto-u.ac.jp \\ http://www.kurims.kyoto-u.ac.jp/ stoimeno/
}

